\title{
Carbon fix
}

\section{Lessons from addiction may help to transform our high-carbon lifestyle.}

It is often said that those of us who live in affluent, developed counties are 'addicted' to our high-carbon lifestyles, which depend on profligate use of energy and material resources. Think of central heating, air conditioning, our reluctance to wean ourselves off international air travel whether for business or pleasure - and the amount of meat that we consume in our diets.

Our love affair with the petrol engine is a case in point. In their advertisements, automobile manufacturers often still portray models of cars as outward symbols of male potency, power and career success, or especially for brands targeted at young women - a penchant for fun and glamour. This is all reminiscent of the way that cigarette brands were once marketed - and to some extent, shamefully, still are in parts of the developing world. Parallels could also be drawn between the way that, in these days of environmental awareness, motor manufacturers increasingly emphasize 'green credentials' such as fuel-efficient engines, with the way that the tobacco industry tried to shift attention to low-tar brands when the evidence that smoking causes lung and other cancers became unassailable.

But it would stretch credulity to push the comparison too far; obviously cars are not 'habit forming' in the sense that cigarettes are, and certainly do not have the addictive characteristics of certain hard drugs. The habit-forming effects of drugs such as nicotine and heroin are mediated by specific receptors in the brain. But it is of course meaningless to talk of a specific 'brain receptor' activated by exposure to a car advert. Nevertheless, it is reasonable to posit that our responses to brands in general, the preferences we develop and the behaviours that we subsequently exhibit as consumers (and more generally as citizens), have a neurobiological basis. This does not at all imply that such preferences and behaviours are deterministically 'fixed', in the sense that they can be modified by learning and experience, or even pharmacologically. If we are sufficiently concerned about the environmental impact of travel, we can by our own volition choose to run an electric car rather than a gas-guzzler.

This seems to be a starting premise of the intriguing new research field of 'neuroconservation', which despite its name has nothing to do with conserving neurons. Rather, as explained by Elisabeth Jeffries (page 776), one of its aims is to study how the brain responds to nature. A working hypothesis is that the way we come to value and appreciate the natural world may involve some of the same brain regions and 'reward' pathways involved in addiction. If that turns out to be the case, which is far from certain, it follows that understanding addiction - both at a neurological and psychological level - might potentially help to devise strategies for shifting people's attitudes in relation to environmental issues such as climate change, thereby promoting climate-friendly behaviours.

\section{Water forecasts}

\section{The effect of climate change on precipitation and flooding is of global concern.}

The strength of water movement through the hydrological cycle is influenced by climate change. Warming of the atmosphere increases its water-holding capacity - by approximately $7 \%$ per degree - which can increase the intensity of precipitation. This impact is expected to be seen predominantly in the tropics and high-latitude regions. Overall precipitation is also expected to increase in the tropics, and it is assumed that wet regions will get wetter and dry areas will get drier.

In a web focus this month on precipitation trends and extremes, we have a collection of articles that looks at the hydrological cycle, climate change and the impacts on society and ecology.

The hydrological cycle has not responded as strongly to climate change as has been predicted. The increase in greenhouse gas concentrations and the warming atmosphere were expected to strengthen the cycle. However, particles in the atmosphere, known as aerosols, are shown by Peili Wu and collaborators to have counteracted the effect of the increased emissions (page 807). From the 1950s higher aerosol concentrations relative to greenhouse gases lead to a suppression of the hydrological cycle. Since the 1980s aerosols have been reduced in the atmosphere, whereas greenhouse gas emissions have continued to increase, resulting in a recovery of the strength of the hydrological cycle. If the current trend continues, the cycle can be expected to intensify.

Changes in total precipitation, as well as in the frequency, intensity, timing and duration will impact human population and infrastructure, as well as ecosystems. Global projections of flooding are made using river discharge and the extent of the area that would be inundated. Yukiko Hirabayashi et al. forecast an increase in flooding in southeast Asia, peninsular India, eastern Africa and the northern Andes, whereas decreased flooding is predicted in some other areas (page 816).

The costs associated with flooding are expected to increase as the frequency and extent of these events rises. In a Letter by Stephane Hallegatte and colleagues, they report the current costs and estimates future costs of coastal flooding (page 802 ), categorized as those owing to socioeconomic change, climate change and land subsidence. In this study they focused on flooding caused by sea-level rise and storms, but the lessons are applicable to other areas where flood protection methods are needed.

The impact of changing precipitation intensity on plant growth is investigated by Andrew Kulmatiski and Karen Beard (page 833). Higher-intensity rainfall will push water deeper into the soil, benefiting plants that have the ability to increase root depth, such as trees and other woody plants.

The supply of water is essential to maintain life, but it is a fine balance as excessive amounts and flooding are dangerous. Better understanding of the hydrological cycle and its changes will help us to adapt to give a healthier future. 